\title{
Editorial: TEN STARS Special Issue
}

This special issue was produced by the TEN-STARS network, an international network of tertiary staff, students, graduates and employers dedicated to furthering research and practice related to graduate employability. The Employability Network, was formed at the 2018 STARS conference (www.unistars.org) in response to shared interests that are very much aligned to those of the Journal of Learning and Teaching for Graduate Employability. It is therefore with great pleasure that we introduce the Journal's first special issue, which is also the network's first collaborative output.

The first issue of the Journal of Teaching and Learning for Graduate Employability was published in 2010, 'as a forum for fostering interdisciplinary dialogue among researchers, teacher scholars, careers staff and industry and professional practitioners concerned about graduate employability' (Quin, 2010). Almost ten years on, dialogue among those key stakeholders is no less important, and assuring graduate employability remains a challenge that spans international contexts. In fact, with growing global uncertainty related to rapid technological developments and the changing world of work, alongside other equally concerning social and political disruptions, the preparation of graduates to face uncertainty in and beyond the workforce is arguably more important than ever (Oliver \& Jorre de St Jorre, 2018). Fortunately, thanks to the dedication of many, and platforms such as this Journal, there is now an expansive and growing body of research and evidence based practice that can be drawn upon - to design, question, and redesign - teaching and learning for graduate employability.

Universities and academics are often at the centre of discourse about graduate employability, but in reality, concerns and responsibilities are shared more broadly amongst: leaders of tertiary institutions (university and non-university providers); academics in and beyond traditional faculty roles; and other staff who contribute to the student experience and graduate outcomes through diverse roles (such as those that contribute to career learning services, learning design, and student support, to name just a few). Students, graduates, employers and professional associations, as well as government and quality assurance agencies, are also key stakeholders, whose influence and perspectives are essential to developing effective strategies (Kinash, Crane, Judd, \& Knight, 2015; Kinash, et al., 2015). With this in mind, the TEN-STARS network was convened by Professor Shelley Kinash, to encourage and support collaboration between all those interested in continuing to discuss, inform, and influence graduate employability. This special issue, was conceived as a way of sharing some of the network's diverse and collective expertise with a broader audience, who are also invited to become part of our network (https://tenstars.graduateemployability.com/).

All of the papers in this TEN STARS Special Issue are empirical, theoretically grounded and richly informed by the growing body of published employability literature. The roles and experience of our contributing authors are diverse, as are their research questions, methods and methodologies. However, all of the inquiries shared have been designed to impact graduate employability, so they are all contextualised to tertiary education, and provide recommendations for further research and efficacious practice related to learning and teaching for employability. Importantly, none of the approaches examined are bolt-ons (requiring already busy students to do more). Rather, authors have focussed on deeply considered approaches to designing and refining the tertiary educational experience, so that employability and careers perspectives are embedded in the student learning experience, and deeply contextualised to disciplines. 
The first three papers, focus on students' experience of employability. These papers examine: what students need to identify who they are, and who they are becoming, in the context of careers.

Bates, Rixon, Carbone and Pilgrim challenge us to think beyond simple notions of employability skills, and to consider what else students will need to develop and pursue their changing career goals. The authors propose that employability is determined by a person's 'professional purpose' which reflects 'commitment to developing a professional future aligned to their personal values, professional aspirations and societal outlook'. The paper draws together findings from two empirical studies examining whether professional purpose is explicit in curriculum purposefully designed to influence students' mindset, one with 33 undergraduate students, and the other with 42 academics. The authors conclude that professional purpose can be made explicit in the curriculum, and propose directions for further research and a conceptual model for the development of professional purpose mindsets.

Whereas Bates et al., propose a model for what students need, Jorre de St Jorre, Elliott, Johnson and Bisset examine students' own priorities and conception of factors relevant to employment and employability. Through analysis of interviews with 138 science students from four Australian universities, the authors investigate whether students are more preoccupied with gaining immediate employment or long-term employability for future careers, and their beliefs about actions that will help them. The authors found that students were most concerned about obtaining employment immediately after graduation. Students were eager to increase their chances of gaining employment and achieving their career goals, but many were unsure about how to proceed. The authors call on universities to 'explicitly design curriculum to help students understand: the career opportunities available; the skills and abilities needed for diverse careers; and the experiences through which they might develop and evidence these'.

Teychenne et al. also examine students' career planning strategies and concerns, and emphasise the need for universities to develop students' career planning skills and behaviours. However, they also illustrate how a brief intervention was able to improve students' career adaptability in a second-year exercise and public health-related unit. The authors' analysed pre- and post-intervention questionnaires completed by 80 students to examine how an online career planning module influenced students career planning, decision making, problem solving/confidence and exploration, and observed small but significant improvements in all areas of career adaptability examined. These results can be interpreted as proof of concept for their career planning approach. This is a space to watch, as the approach described is expanded and tested across more diverse groups of learners, including those in other disciplines.

The remaining papers shift in focus from students' experience of employability, to those of other stakeholders. The first three of these, examine challenges and opportunities associated with developing graduate employability at scale.

Like other papers already described, Bridgstock, Grant-Iramu and McAlpine emphasise the importance of career development learning and encourage institutions to integrate it with other curriculum so that all students can benefit. However, the authors explore the challenges and opportunities faced by tertiary institutions seeking to integrate career development learning across the curriculum. The authors draw on case studies and interviews with 37 staff across nine universities (in Australia and internationally), and present three cases to illustrate a range of policies and practices that exist, and their own strategy recommendations. The authors point to the importance of cross-disciplinary collaboration between career development practitioners, learning designers and academic units to embedding career development learning into the curriculum at scale.

Young et al. also highlight the importance of cross-disciplinary collaboration to embedding learning relevant to employability into the curriculum. In their paper, Assessment-led reform: Creating a sustainable culture for on and off campus WIL, the authors describe an action research project used to enhance assessment across two science programs at an Australian

Kinash, S., \& Jorre de St Jorre, T. (2019). Editorial: TEN STARS Special Issue. Journal of Teaching and Learning for Graduate Employability, 10(1), 1-6. 
University. The authors utilised a mixed method approach to collecting and analysing data from students, industry and course teams, to identify factors that were important to redesigning assessment at a program level. In particular, they highlight the importance of building the confidence of discipline experts and developing transparent partnerships between students, industry and faculty.

Dollinger and Brown propose a model that institutions might use to guide comparison of different work-integrated learning strategies. The authors utilise student and staff interviews to compare the benefits and drawbacks associated with four different types of work-integrated learning, implemented across three Australian universities. The authors provide a comparison table, which succinctly plots low, medium or high ease of implementation, barriers, scalability, authenticity and proximity. The paper provides further evidence to support the inter-textual claim that WIL is an efficacious employability strategy. The type of WIL that appears to hold the most promise, according to the authors' cost-benefit analysis, was industry-based placements within formal subjects. However, the authors acknowledge that their sample sizes are low, and that their findings should be interpreted as postulations or hypotheses. This highlights opportunities, for these authors or others, to replicate and extend this research and analysis.

The next three papers extend earlier discussion about the value of collaboration and industry partnerships, by examining how universities might deepen industry engagement and partnerships to design and deliver curriculum that improves graduate outcomes.

Male and King examine how industry engagement contributes to graduate learning outcomes and employability in engineering. The authors draw on the results of a national study that examined student and employer perspectives of engineering education. They present a theoretical framework and model for designing effective engineering education, and guidelines for effective industry engagement, that include recommendations for faculties, industry, professional and industry peak bodies, and governments. The authors also track the long-term development of WIL in engineering and flag a change in the peak professional body's conceptualisation of the role of WIL, from student 'exposure' to student 'engagement'. While this inquiry is situated in engineering, it provides useful examples and a benchmark for other disciplines.

Walker, Morrison and Hay also extol the virtues of professional experience for developing employability. In the context of teacher preparation, they examine how and to what extent strategic partnerships influence the quality of interactions between a School of Education and partner schools, including students perceptions of their in-school placement experiences. The researchers analysed survey responses from pre-service teachers who had undertaken placements at partnership $(n=25)$ and non-partnership schools $(n=26)$. Over $90 \%$ of students in both cohorts agreed that the 'final practicum experience was a valuable part' of their teacher preparation, but there were subtle differences between students experience in partnership and non-partnership schools. Overall the partnership program had positive impacts on pre-service teacher preparation, however, an unanticipated finding was that preservice teachers in non-partner school settings actually had more opportunities to observe and interact with other teachers (not just their supervising mentor) and participate in broader school activities. Thus the study highlights the importance of ongoing partnership development informed by evaluation of student and stakeholder experiences.

Clarke and Windslade affirm the findings of Walker, et al. described immediately above, but provide an alternative model for developing school-university partnerships in teacher education. 'Initial teacher education programs have been perennially criticised by school educators for their lack of connection to the authenticity of 'real-world' classroom practice'. The authors seek to address this through the development of a team teaching approach collaboratively designed by an academic experienced in teacher education and two secondary school teachers. Although the pilot project described was implemented at a small scale, the researchers collected and analysed data from multiple sources to identify common themes, 
including: interviews with teacher partners pre- and post- teaching the professional experience subject; student blogs and experience surveys; and journaling by the academic. The authors identify challenges and benefits associated with the partnership model, but overall found that it added value to the professional experience of students, while also having reciprocal value for partner school teachers. A key takeaway from this paper is the importance of respect for different expertise to balancing power and building effective and reciprocal partnerships. The trial has established proof of concept and highlighted areas for further development, in particular expansion at scale. While situated in teacher education, the paper provides an efficacious employability initiative that could be adapted to other contexts, disciplines and industries.

The final three papers, each expand discussion about what tertiary institutions are doing, and could do, to support graduate employability in unique ways.

Fraser, Duignan, Stewart and Rodrigues expand the context of this special issue, both in terms of geography and tertiary institution type. Their inquiry across is set across seven New Zealand Institutes of Technology and Polytechnics (ITPs). The researchers used classroom observation and interviews with 23 experienced ITP teachers to identify practices used to nurture ten core employability attributes in students and graduates: positive attitude, communication, teamwork, self-management, willingness to learn, thinking skills, resilience, innovation, entrepreneurship and cultural competence. A key contribution of the authors' is an open, online dashboard of strategies and resources organised and tagged by the ten employability attributes (https://sites.google.com/view/employabilityskills). The authors conclude their paper by writing, "we have only just begun." Readers are encouraged to connect with the authoring team and build-upon this action research.

Oraison, Konjarski and Howe, take an important step back from the strategies used to develop employability skills, to examine whether the skills articulated are actually fit for purpose. The authors examine alignment between course specific graduate attributes, accreditation requirements and industry employability criteria (selection criteria in job advertisements) across three disciplines at an Australian University. In other words, they asked - are the capabilities that graduates are expected to develop (course specific graduate attributes) equivalent to what professional bodies say graduates need (accreditation requirements) and what employers are actually asking for in job advertisements (industry employability criteria)? The authors found that the answer to their question was 'mostly'. However, universityarticulated graduate attributes and professional association accreditation standards both tend to include 'cultural understandings' and 'attitudes towards inclusion and diversity', whereas the selection criteria associated with job advertisements seldom include these components. Does one or the other side of the equation need to change? Is this a case in which universities and professional associations are trail-blazers and that employers will follow this lead? Read this compelling paper to inform your reflections.

Finally, this special issue concludes with a novel paper examining universities as employers of graduates. Arvanitakis et al., peer into our own backyard, and inquire into how well universities are doing at supporting our graduates to transition into and through university careers, in particular, work related to learning and teaching. The five authors candidly share and analyse their own career stories, alongside 322 recent university vacancies from two universities, to investigate: what do learning and teaching careers look like at universities? And, what are the occupational patterns, satisfactions and concerns of staff in those careers? They conclude that the transition into and through university careers in learning and teaching is mostly haphazard, and present recommendations for universities, and graduates pursuing or navigating careers in higher education teaching and learning.

In summary, this Special Issue of the Journal of Teaching and Learning for Graduate Employability includes twelve empirical investigations into the Scholarship of Teaching and Learning for heightened graduate employability. The included inquiries span diverse contexts and approaches, from large scale national projects to small action research projects, and 
cross-disciplinary inquiries to studies that focus-in on particular disciplines such as science, engineering and teacher preparation. However, all of the studies included have been selected because separately, but especially together, they build on the existing literature and approaches for developing graduate employability, and provide value able insights that can be adopted in similar contexts or adapted to enrich others.

\section{Acknowledgements}

We would like to thank all of the TEN-STAR members who contributed to this special issue, from those who helped to conceive the idea, to those who wrote and reviewed papers. We also offer our sincere thanks to Prof Beverley Oliver and the JLTGE editorial board for their support. Last but not least, we would like to acknowledge the guidance, expertise and dedication of Journal Manager, Di Gardiner, who has been integral to the publication of this special issue.

\section{Guest Editors: Professor Shelley Kinash and Dr Trina Jorre de St Jorre}




\section{References}

Kinash, S., Crane, L., Judd, M-M., \& Knight, C. (2015). Discrepant stakeholder perspectives on graduate employability strategies. Higher Education Research \& Development, 35(5), 951967. doi:10.1080/07294360.2016.1139555

Kinash, S., Crane, L., Judd, M-M., Mitchell, K., McLean, M., Knight, C., ... Schulz, M. (2015). Supporting graduate employability from generalist disciplines through employer and private institution collaboration. Report prepared for the Office for Learning and Teaching. Canberra: Australian Government.

Oliver, B., \& Jorre de St Jorre, T. (2018). Graduate attributes for 2020 and beyond:

Recommendations for Australian higher education providers. Higher Education Research \& Development, 37, 821-836.

Quin, R. (2010). Editorial. Journal of Teaching and Learning for Graduate Employability, 1, 2-12. 